\title{
Three-dimensional effect on the effective thermal conductivity of porous media
}

\author{
Moran Wang ${ }^{1}$, Jinku Wang ${ }^{2}$, Ning Pan ${ }^{1}$, Shiyi Chen ${ }^{3}$ and \\ Jihuan $\mathrm{He}^{4}$ \\ ${ }^{1}$ Department of Biological and Agricultural Engineering, University of California, Davis, \\ CA 95616, USA \\ ${ }^{2}$ School of Aerospace, Tsinghua University, Beijing 100084, People's Republic of China \\ ${ }^{3}$ Department of Mechanical Engineering, Johns Hopkins University, Baltimore, MD 21218, \\ USA \\ ${ }^{4}$ College of Science, Donghua University, Shanghai 200051, People's Republic of China \\ E-mail: mmwang@ucdavis.edu
}

Received 6 September 2006, in final form 20 October 2006

Published 15 December 2006

Online at stacks.iop.org/JPhysD/40/260

\begin{abstract}
A three-dimensional mesoscopic method is developed for predicting the effective thermal conductivity of multiphase random porous media. The energy transport equations are solved by a lattice Boltzmann method for multiphase conjugate heat transfer through a porous structure whose morphology is characterized by a random generation-growth algorithm. Our numerical results show that the cell number in the third dimension influences the resulting effective thermal conductivity of three-dimensional porous media. The predicted effective thermal conductivity varies with the cell number in the third dimension following an exponential relationship, and it requires in the examples at least 10 cells along the third dimension before the predictions stabilize. Comparisons with the experimental data show that the effective thermal conductivities measured by the hot-probe and hot-wire techniques agree well with the predicted results by the two-dimensional model, whereas those measured by the transient comparative method agree more with the three-dimensional predictions.
\end{abstract}

\section{Introduction}

For better understanding of transport mechanism in soils, rocks and other engineering materials, the effective thermal conductivity of porous media has been studied for well over one hundred years in both theory and experiment [1-3]. Recently, it has gained more attention due to its new applications in functional material design, MEMS devices cooling techniques, textile and food engineering and even human medical technologies [4-7].

In experiments, the hot-wire and hot-probe are the most popular techniques for local thermal conductivity measurement for soft matter, which are based on a linear heat source and an axis-symmetric measurement system [8,9]. They have been used for measuring the thermal conductivities of soils, foods and even liquids [10-17]. In high porosity solid structure cases, however, the parallel plate and hot plate have been more frequently used [18-22]. Recently, Carson et al [23] developed a 'comparative method' to measure the thermal conductivity in highly non-homogeneous cases.

To model the effective thermal conductivity of porous media, the semi-empirical theoretical models often used are generally based on network combinations of the series and parallel models [24-29]. Wang et al [30] also presented simple combinatory rules for complex materials. Owing to the rapid developments in computer and computational techniques in the past twenty years, pure numerical modelling methods have been used to predict the thermal conductivity of porous media [31-43]. Thovert et al [31] calculated the thermal conductivity of random media and regular fractals by solving the Laplace equation using the finite-difference method. Bakker [32] determined the thermal conductivity of porous media through the finite element method. However, 
the traditional partial difference equation (PDE) solvers often impose huge or even prohibitive computational cost when the porous structure is too complicated, especially when the fluid-solid conjugate problem is considered. Therefore the stochastic-statistic methods for transport in porous media have gained increasing attention in recent years [33, 34]. Shoshany et al [35] and Barta and Dieska [36] modelled the thermal conductivity of porous materials using the Monte Carlo methods. Zhang et al [37,38] developed a randomly mixed model (RMM) to predict the effective thermal conductivity of moist porous media. However, Qian et al [39] used a twodimensional five-speed (D2Q5) lattice Boltzmann model to simulate the effective thermal conductivity of porous materials with no fluid-solid conjugate heat transfer considered. Wang et al [40] built up a full stochastic-statistic approach for the effective thermal conductivity predictions of random porous media, which include a two-dimensional nine-speed (D2Q9) lattice Boltzmann method (LBM) for fluid-solid conjugate heat transfer [41] and a random generation-growth method to generate morphology of multiphase random porous media. Wang's predictions agreed rather well with a series of experimental data.

Based on the previous work, this paper will develop a three-dimensional stochastic-statistic based method for predicting the effective thermal conductivity of porous media. The results will be compared with the two-dimensional predictions and the existing experimental data. The threedimensional effect of the effective thermal conductivity of porous media will therefore be discussed.

\section{Numerical method}

\subsection{Lattice Boltzmann algorithm}

The LBM is intrinsically a mesoscopic approach based on the evolution of statistical distribution on lattices and has achieved considerable success in simulating fluid flows and associated transport phenomena [42-44]. The most important advantages of the LBM are the easy implementation of multiple intercomponent interactions and complex geometry boundary conditions $[45,46]$. Conservations can hold automatically in general without additional computational efforts $[47,48]$. The thermal models for the two-dimensional and three-dimensional LBM have been developed recently [49-51]. Here we have adapted our previous lattice Boltzmann algorithm for fluidsolid conjugate heat transfer [41] into the three-dimensional mode.

For the pure thermal conduction in porous media, the temperature evolution equation in either the fluid or the solid phase can be generally given as

$g_{\alpha}\left(\boldsymbol{r}+\boldsymbol{e}_{\alpha} \delta_{t}, t+\delta_{t}\right)-g_{\alpha}(\boldsymbol{r}, t)=-\frac{1}{\tau_{g}}\left[g_{\alpha}(\boldsymbol{r}, t)-g_{\alpha}^{\mathrm{eq}}(\boldsymbol{r}, t)\right]$,

where $g_{\alpha}^{\mathrm{eq}}$ is the equilibrium distribution in each direction, $\boldsymbol{e}_{\alpha}$ the discrete lattice velocity and $\tau_{g}$ the dimensionless relaxation time for each phase. For a D2Q9 model, they are

$$
\begin{gathered}
g_{\alpha}^{\mathrm{eq}}= \begin{cases}0 & \alpha=0 \\
\frac{1}{6} T & \alpha=1 \text { to } 4 \\
\frac{1}{12} T & \alpha=5 \text { to } 8,\end{cases} \\
\boldsymbol{e}_{\alpha}= \begin{cases}(0,0) & \alpha=0 \\
( \pm 1,0) c,(0, \pm 1) c & \alpha=1 \text { to } 4 \\
( \pm 1, \pm 1) c & \alpha=5 \text { to } 8\end{cases}
\end{gathered}
$$

and

$$
\tau_{g}=\frac{3}{2} \frac{k}{c^{2} \delta_{t}}+0.5
$$

while for a D3Q15 model they are

$g_{\alpha}^{\mathrm{eq}}= \begin{cases}0 & \alpha=0 \\ \frac{1}{9} T & \alpha=1 \text { to } 6 \\ \frac{1}{24} T & \alpha=7 \text { to } 14,\end{cases}$

$\boldsymbol{e}_{\alpha}= \begin{cases}(0,0,0) & \alpha=0 \\ ( \pm 1,0,0) c,(0, \pm 1,0) c,(0,0, \pm 1) c & \alpha=1 \text { to } 6 \\ ( \pm 1, \pm 1, \pm 1) c & \alpha=7 \text { to } 14\end{cases}$

and

$$
\tau_{g}=\frac{9}{5} \frac{k}{c^{2} \delta_{t}}+0.5
$$

where $\delta_{t}$ is the time step, $k$ the thermal conductivity for each phase and $c$ a pseudo sound speed whose value can take any positive value theoretically only to insure the $\tau_{g}$ value within $(0.5,2)[41,46]$. The temperature and the heat flux can then be calculated according to [52]

$$
\begin{gathered}
T=\sum_{\alpha} g_{\alpha}, \\
q=\left(\sum_{\alpha} \boldsymbol{e}_{\alpha} g_{\alpha}\right) \frac{\tau_{g}-0.5}{\tau_{g}} .
\end{gathered}
$$

After the temperature field is solved, the effective thermal conductivity, $k_{\text {eff }}$, can be determined:

$$
k_{\mathrm{eff}}=\frac{L \cdot \int q \cdot \mathrm{d} A}{\Delta T \int \mathrm{d} A},
$$

where $q$ is the steady heat flux through the media cross section area $\mathrm{d} A$ between the temperature difference $\Delta T$ with a distance $L$.

\subsection{Boundary conditions}

For the isothermal boundary treatment, we follow the bounceback rule of the non-equilibrium distribution proposed by Zou and $\mathrm{He}$ [53]:

$$
g_{\alpha}-g_{\alpha}^{\mathrm{eq}}=-\left(g_{\beta}-g_{\beta}^{\mathrm{eq}}\right),
$$

where $\alpha$ and $\beta$ represent opposite directions, and the equilibrium distribution can be calculated based on the local boundary temperature. 
Table 1. Predictions of effective thermal conductivities for two kinds of structures.

\begin{tabular}{|c|c|c|c|c|}
\hline \multirow[b]{2}{*}{ Results $c\left(\mathrm{~m} \mathrm{~s}^{-1}\right)$} & \multicolumn{2}{|c|}{ Parallel mode } & \multicolumn{2}{|c|}{ Series mode } \\
\hline & Predictions $\left(\mathrm{W} \mathrm{m}^{-1} \mathrm{~K}^{-1}\right)$ & Deviations (\%) & Predictions $\left(\mathrm{W} \mathrm{m}^{-1} \mathrm{~K}^{-1}\right)$ & Deviations (\%) \\
\hline 100 & 1.26267491 & 0.0139 & 0.04950495 & 0.0000 \\
\hline 1000 & 1.26244815 & -0.0058 & 0.04950495 & 0.0000 \\
\hline 10000 & 1.26256610 & 0.0052 & 0.04950495 & 0.0000 \\
\hline
\end{tabular}

where $k_{1}=2.5 \mathrm{~W} \mathrm{~m}^{-1} \mathrm{~K}^{-1}, k_{2}=0.025 \mathrm{~W} \mathrm{~m}^{-1} \mathrm{~K}^{-1}$.

For the insulated boundary, we tried the Neumann boundary treatment $[41,52]$ and let the boundary temperature gradient be equal to zero. To prevent heat flux from leaking along the insulate surfaces, a specular reflection treatment is implemented. It has been proved that the current boundary treatments have the second order accuracies [53].

\section{Results and discussion}

The proposed method will be used to predict the effective thermal conductivity of the three-dimensional porous media in this section. First of all, the method is validated by comparing with the theoretical solutions for two simple structures. The three-dimensional predictions with proper simulation parameters will then be compared against the twodimensional cases and experimental data so that the third dimension influence on the effective thermal conductivity of porous media is discussed.

\subsection{Benchmarks}

To validate the algorithm and the codes, the numerical predictions are compared with the theoretical solutions for two hypothetical structure cases: parallel mode and series mode. The structures are formed in two phases and the thermal conductivities are $2.5 \mathrm{~W} \mathrm{~m}^{-1} \mathrm{~K}^{-1}$ and $0.025 \mathrm{~W} \mathrm{~m}^{-1} \mathrm{~K}^{-1}$, respectively. The fractions of the two phases are equal so that the theoretical solution of the effective thermal conductivity is $1.2625 \mathrm{~W} \mathrm{~m}^{-1} \mathrm{~K}^{-1}$ for parallel mode and $0.04950495 \mathrm{~W} \mathrm{~m}^{-1} \mathrm{~K}^{-1}$ for series mode. Table 1 lists our predictions of the effective thermal conductivities and the deviations from the simple theoretical solutions as functions of the pseudo sound speed $c$. It is clear that our predictions agree perfectly well with the theoretical solutions with the maximum deviation less than $0.02 \%$ when $c$ changes from 100 to $10000 \mathrm{~m} \mathrm{~s}^{-1}$. The results validate the proposed algorithm and the boundary implements. A larger $c$ leads to a more accurate prediction yet requires a higher computational cost. In the following simulations, $c$ is set to $1000 \mathrm{~m} \mathrm{~s}^{-1}$ unless specified otherwise.

\subsection{Randomly generated porous media}

To bring the random characteristics of natural porous media into modelling, the random effect has to be introduced during the generation of porous media structure. The random location of obstacles is the most popular method to construct an artificial porous medium [35-38]. Coveney et al [54] proposed a pore growth-with-time model to control the pores size and connectivity. Wang et al [40] presented a full description of the structure generation method, termed the quartet structure
Table 2. Predicted effective thermal conductivities for different core distribution probabilities.

\begin{tabular}{lll}
\hline$c_{\mathrm{d}}$ & $k_{\mathrm{eff}}\left(\mathrm{W} \mathrm{m}^{-1} \mathrm{~K}^{-1}\right)$ & $k_{\mathrm{eff}} / k_{\mathrm{eff}, c_{\mathrm{d}}=0.3}$ \\
\hline 0.001 & 1.501 & 0.9311 \\
0.01 & 1.519 & 0.9423 \\
0.1 & 1.592 & 0.9876 \\
0.3 & 1.612 & 1.0000 \\
\hline
\end{tabular}

where $k_{s}=2.50 \mathrm{~W} \mathrm{~m}^{-1} \mathrm{~K}^{-1}, k_{g}=0.025$ $\mathrm{W} \mathrm{m}{ }^{-1} \mathrm{~K}^{-1}$.

generation set (QSGS), for generating micro-morphology of random porous media based on the cluster growth theory [55]. The QSGS method has been used in two-dimensional simulations and has shown good agreement with a series of experimental data. Here we implement the QSGS method in three-dimensional isotropic systems.

To distinguish the present QSGS method from the previous random obstacle location method [38], the effect of core distribution probability, $c_{\mathrm{d}}$, on the effective thermal conductivity is studied. Consider the gas-solid porous media at a porosity $\varepsilon=0.7$. The solid cores grow by the QSGS method with a fraction of 0.3 . When $c_{\mathrm{d}}$ equals 0.3 , the QSGS method will become equivalent to the random obstacle location method. Once $c_{\mathrm{d}}$ is smaller than 0.3 , each solid core will have a three-dimensional structure, not just a cube.

Table 2 shows our predicted effective thermal conductivities versus the core distribution probability, $c_{\mathrm{d}}$, at $k_{s}=$ $2.50 \mathrm{~W} \mathrm{~m}^{-1} \mathrm{~K}^{-1}$ and $k_{g}=0.025 \mathrm{~W} \mathrm{~m}^{-1} \mathrm{~K}^{-1}$. The results reveal that the effective thermal conductivity of porous media decreases with the core distribution probability. When $c_{\mathrm{d}}$ changes from 0.3 to 0.01 , the effective thermal conductivity decreases by over $5 \%$. It is indicated that the influence of porous structures on the effective thermal conductivity should be taken into consideration if the pore size is not negligible.

\subsection{Influence of the third dimension}

The three-dimensional transport phenomena often differ from the two-dimensional cases under the same boundary conditions due to the different surface-to-volume ratio [56,57]. Wang and $\mathrm{Li}$ [58] compared the gas flows in two- and three-dimensional microchannels and found an exponential relationship between the velocities in a three-dimensional channel and in a twodimensional channel. Here we perform the effective thermal conductivity predictions in both two- and three-dimensions under the same boundary conditions and with the same simulation parameters. We maintain the identical length and height of the 2D cell domains but add the cell number along the third dimension to set up a 3D case. Comparisons between the three-dimensional results for different cell numbers in the 


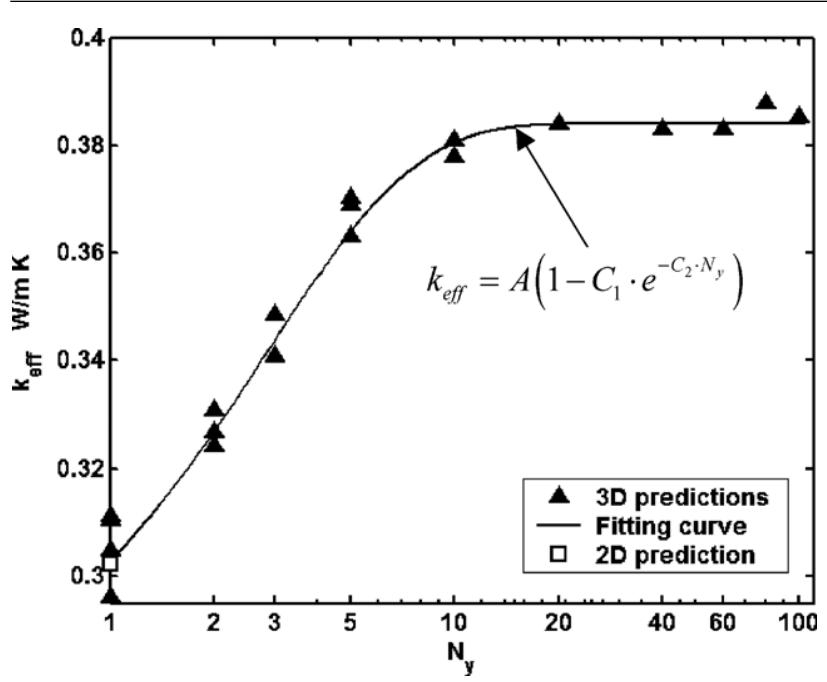

Figure 1. The predicted effective thermal conductivities of three-dimensional two-phase porous media versus the cell numbers $N_{y}$ in the third direction. The simulation parameters are $k_{s}=0.60 \mathrm{~W} \mathrm{~m}^{-1} \mathrm{~K}^{-1}, k_{g}=0.035 \mathrm{~W} \mathrm{~m}^{-1} \mathrm{~K}^{-1}, \varepsilon=0.3$ and $c_{\mathrm{d}}=0.01$. The fitted parameters are $A=0.384, C_{1}=0.30$ and $C_{2}=0.35$.

third dimension and the two-dimensional results will indicate the three-dimensional effects.

The two-phase porous media are generated at $\varepsilon=$ $0.3, c_{\mathrm{d}}=0.01$ and the simulation parameters are $k_{s}=$ $0.60 \mathrm{~W} \mathrm{~m}^{-1} \mathrm{~K}^{-1}$ and $k_{g}=0.035 \mathrm{~W} \mathrm{~m}^{-1} \mathrm{~K}^{-1}$. The grids for the two-dimensional predictions are set as $N_{2} \times N_{2}$, where $N_{2}=200$, and for the three-dimensional predictions as $N_{3} \times N_{y} \times N_{3}$, where a typical value of $N_{3}$ is normally 200 . We have changed the values of $N_{3}$ from 60 to 300, and the results remain almost the same. For the current grid arrangements, the discretization errors are smaller than $3 \%$ caused by the random structures.

Figure 1 shows the impact of the third dimension cell numbers $N_{y}$ on the effective thermal conductivity predictions. The two-dimensional result is plotted in the same figure, which can be viewed as a special case of the three-dimensional result at $N_{y}=1$. The three-dimensional effective thermal conductivity increases sharply with the third dimension cell number when $N_{y}<10$ and then levels off for larger $N_{y}$. Similar to [58], a similar exponential relationship is obtained by fitting the predictions as shown in figure 1 . For the specific simulation case, the fitted parameters are $A=0.384$, $C_{1}=0.30, C_{2}=0.35$.

The results indicate the following: (i) the predicted effective thermal conductivities are always smaller at twodimensional cases than at three-dimensional ones under the same boundary conditions and simulation parameters, which is consistent with the previous work of Kirkpatrick based on the effective medium theory [59], (ii) the cell number in the third dimension has to be at least over a certain number $(=10$ in this case) to stabilize the influence of the third dimension and (iii) once the cell number in the third dimension is large enough, the predicted effective thermal conductivity becomes independent of the cell number $N_{y}$.

Furthermore, the ratios of the predicted effective thermal conductivities between $2 \mathrm{D}$ and $3 \mathrm{D}$ are also related to the porosities. Figure 2 depicts the relationship by using the

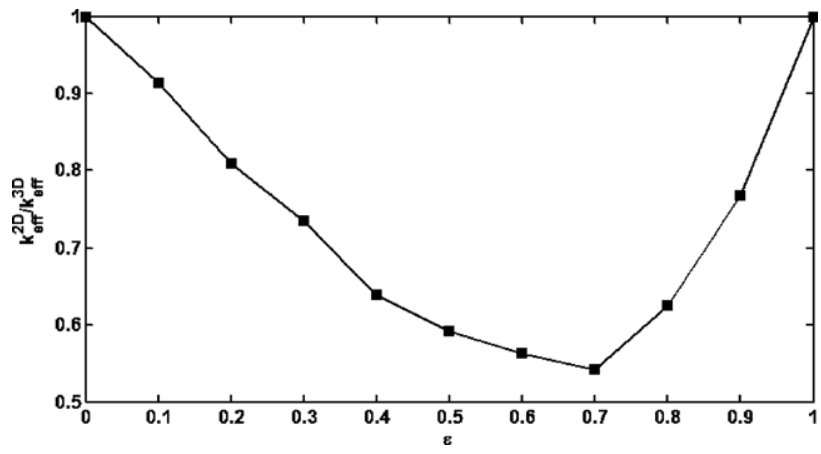

Figure 2. The ratios of two-dimensional to three-dimensional predicted effective thermal conductivities for different porosities of three-dimensional two-phase porous media. The simulation parameters are $k_{s}=0.60 \mathrm{~W} \mathrm{~m}^{-1} \mathrm{~K}^{-1}, k_{g}=0.035 \mathrm{~W} \mathrm{~m}^{-1} \mathrm{~K}^{-1}$ and $c_{\mathrm{d}}=0.01$.

simulation parameters as $k_{s}=0.60 \mathrm{~W} \mathrm{~m}^{-1} \mathrm{~K}^{-1}, k_{g}=$ $0.035 \mathrm{~W} \mathrm{~m}^{-1} \mathrm{~K}^{-1}$ and $c_{\mathrm{d}}=0.01$. The results indicate again that the two-dimensional predictions are always smaller than the three-dimensional ones unless the porosity $\varepsilon$ equals zero or unity. The ratio does not vary with the porosity monotonically and reaches a minimum value, in this case, smaller than 0.6 when the porosity $\varepsilon \approx 0.7$.

\subsection{Comparisons with experimental data}

The present two- and three-dimensional predictions are also compared with the existing experimental data. As reviewed in the introduction, the hot-probe is a convenient measurement for the effective thermal conductivity in soft matter; however since it is based on a line heat source and axial symmetric assumption, this method is generally regarded as a twodimensional technique [60]. Wang et al [40] have shown that a series of experimental data measured by the hot-probe or hot-wire methods agree well with their two-dimensional predictions. Here we compare the measured effective thermal conductivities of moist sands under frozen and unfrozen states $[11,12]$ with the corresponding predictions in figure 3 . The parameters in both two- and three-dimensional calculations are $\varepsilon=0.52, k_{s}=2.85 \mathrm{~W} \mathrm{~m}^{-1} \mathrm{~K}^{-1}, k_{w}=0.5924 \mathrm{~W} \mathrm{~m}^{-1} \mathrm{~K}^{-1}$, $k_{g}=0.0249 \mathrm{~W} \mathrm{~m}^{-1} \mathrm{~K}^{-1}$ and $k_{\text {ice }}=2.38 \mathrm{~W} \mathrm{~m}^{-1} \mathrm{~K}^{-1}$ adopted from [61]. The two-dimensional simulations use a $200 \times 200$ grid, and the three-dimensional cases use a $60 \times 60 \times 60$ grid for acceptable computational costs. The results show that the experiment data are consistent with the two-dimensional predictions and yet deviate much from the three-dimensional predictions.

We also notice that Zhang's recent three-dimensional random simulations also exhibit good agreement with the experimental data used [38]. Based on the analysis above, the reason for such agreement might be as follows: (i) the cell number in the third dimension is not large enough so that the three-dimensional simulations are actually close to the two-dimensional ones and (ii) the RMM did not consider the influence of the pore structure, which was actually a special case at $c_{\mathrm{d}}=\varepsilon$.

Carson et al [23] developed a transient comparative method to measure the effective thermal conductivity of a pseudo-porous food analogue. There was no two-dimensional 


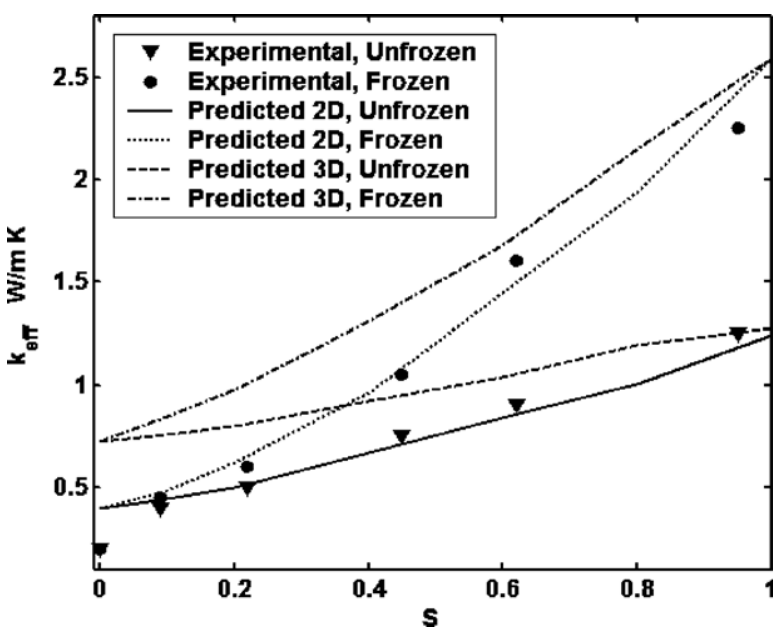

Figure 3. Comparisons between our numerical predictions with the experimental data of Singh et al [12] measured by the hot-probe method.

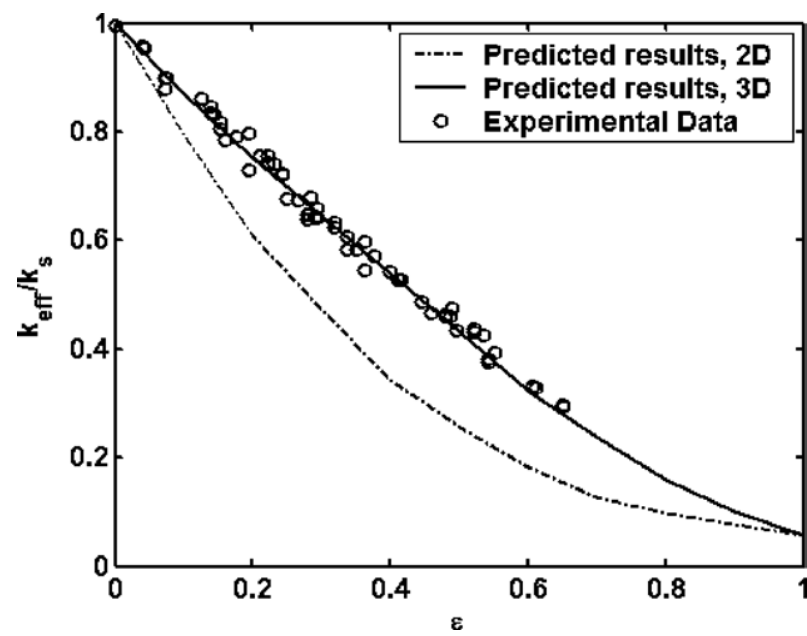

Figure 4. Comparisons between our numerical predictions and the experimental data of Carson et al [23] measured by a transient comparative method.

assumption in this measurement technique. Figure 4 shows the comparison of experimental data with the respective two-dimensional and three-dimensional predictions using the present models. The used parameters are $c_{\mathrm{d}}=0.01, k_{s}=$ $0.60 \mathrm{~W} \mathrm{~m}^{-1} \mathrm{~K}^{-1}$ and $k_{g}=0.035 \mathrm{~W} \mathrm{~m}^{-1} \mathrm{~K}^{-1}$. Again the two-dimensional simulations use $200 \times 200$ grids and the three-dimensional cases use $60 \times 60 \times 60$ grids. This time, however, the three-dimensional predictions are closer to the experimental data, while the two-dimensional predictions are much lower. Although the generated random structures are not exactly the same as those in Carson's experiments, which might lead to possible differences [62], this comparison may still serve as evidence to show that Carson's transient comparative method is a three-dimensional measurement technique.

\section{Conclusions}

The three-dimensional effect on the effective thermal conductivity predictions of porous media has been investigated numerically using a stochastic-statistic based method.
Compared with the two-dimensional results under the same boundary conditions and parameters, the three-dimensional predictions show a dependence on both the generated structure and the cell number in the third dimension. The threedimensional predictions increase with the third dimension cell number by an exponential relationship. The cell number in the third dimension has to be greater than a certain value to eliminate the influence and the predicted effective thermal conductivity then becomes independent of the cell number in the third dimension.

For given porosities, the two-dimensional predicted effective thermal conductivity is always smaller than the three-dimensional result unless the porosity equals zero or unity. The ratio of the two reaches a minimum value at a certain porosity level. Comparisons between the numerical predictions and the experimental data show that the hotprobe and hot-wire based methods are two-dimensional measurement techniques, whereas the transient comparative method is more applicable to three-dimensional situations. The methods and analyses presented in this work can be generalized for various heterogeneous materials.

\section{Acknowledgments}

The present work is supported by the National Textile Center (NTC) M04-CD01. The authors would also like to acknowledge the helpful discussions with Dr J F Zhang and Dr J K Carson.

\section{References}

[1] Muskat M and Wyckoff R D 1937 The Flow Of Homogeneous Fluids Through Porous Media (New York: McGraw-Hill)

[2] Ghildyal B P and Tripathi R P 1973 Soil Physics (London: Wiley)

[3] Zimmerman R W 1991 Compressibility of Sandstones (New York: Elsevier)

[4] Ingham D B, Bejan A, Mamut E and Pop I 2003 Emerging Technologies and Techniques in Porous Media (London: Kluwer)

[5] Sayari A and Jaroniec M 2005 Nanoporous Materials IV (New York: Elsevier)

[6] Ingham D B and Pop I 2005 Transport Phenomena in Porous media III (Oxford: Elsevier)

[7] Pan N and Zhong W 2006 Fluid Transport Phenomena in Fibrous Materials (Cambridge, UK: Woodhead Publishing)

[8] Blackwell J H 1954 A Transient-flow method for determination for thermal constants of insulating materials in bulk J. Appl. Phys. 25 137-44

[9] Blackwell J H 1956 The axial-flow error in the thermal-conductivity probe Can. J. Phys. 34 412-17

[10] Carslaw H S and Jaeger J C 1959 Conduction of Heat in Solids (Oxford: Clarendon)

[11] Sepaskhah A R and Boersma L 1979 Thermal-conductivity of soils as a function of temperature and water-content Soil Sci. Soc. Am. J. 43 439-44

[12] Singh A K, Singh R, Chaudhary D R 1990 Prediction of effective thermal conductivity of moist porous materials J. Phys. D: Appl. Phys. 23 698-702

[13] Liang X G, Ge X S, Zhang Y P, Wang G J A 1991 convenient method of measuring the thermal-conductivity of biological tissue Phys. Med. Biol. 36 1599-605

[14] Liang X G 1995 The boundary induced error on the measurement of thermal-conductivity by transient hot-wire method Meas. Sci. Technol. 6 467-71 
[15] Liang X G, Zhang Y P and Ge X S 1999 The measurement of thermal conductivities of solid fruits and vegetables Meas. Sci. Technol. 10 N82-6

[16] Zhang H F, He L Q, Cheng S X, Zhai Z T and Gao D Y 2003 A dual-thermistor probe for absolute measurement of thermal diffusivity and thermal conductivity by the heat pulse method Meas. Sci. Technol. 14 1396-401

[17] Zhang H F, Zhao G, Ye H, Ge X S and Cheng S X 2005 An improved hot probe for measuring thermal conductivity of liquids Meas. Sci. Technol. 16 1430-5

[18] Rahman S 1995 Food Properties Handbook (Boca Raton, Fl: CRC Press)

[19] Liang X G and Qu W L 1997 An improved single-plate method for measuring the thermal resistance of fibrous materials Meas. Sci. Technol. 8 525-9

[20] Calmidi V V and Mahajan R L 1999 The effective thermal conductivity of high porosity fibrous metal foams $J$. Heat Transfer ASME 121 466-71

[21] Paek J W, Kang B H, Kim S Y and Hyun J M 2000 Effective thermal conductivity and permeability of aluminum foam materials Int. J. Thermophys. 21 453-64

[22] Bhattacharya A, Calmidi V V and Mahajan R L 2002 Thermophysical properties of high porosity metal foams Int. J. Heat Mass Transfer 45 1017-31

[23] Carson J K, Lovatt S J, Tanner D J and Cleland A C 2004 Experimental measurements of the effective thermal conductivity of a pseudo-porous food analogue over a range of porosities and mean pore sizes J. Food Eng. 63 87-95

[24] Bouguera A 1999 Prediction of effective thermal conductivity of moist wood concrete J. Phys. D: Appl. Phys. 32 1407-14

[25] Liang X G and Qu W 1999 Effective thermal conductivity of gas-solid composite materials and the temperature difference effect at high temperature Int. J. Heat Mass Transfer. 42 1885-93

[26] Staggs J E J 2002 Estimating the thermal conductivity of chars and porous residues using thermal resistor networks Fire Safety J. 37 107-19

[27] Singh R and Kasana H S 2004 Computational aspects of effective thermal conductivity of highly porous metal foams Appl. Therm. Eng. 24 1841-9

[28] Ma Y T, Yu B M, Zhang D M and Zou M Q 2004 Fractal geometry model for effective thermal conductivity of three-phase porous media J. Appl. Phys. 95 6426-34

[29] Carson J K, Lovatt S J, Tanner D J and Cleland A C 2006 Predicting the effective thermal conductivity of unfrozen, porous foods J. Food Eng. 75 297-307

[30] Wang J F, Carson J K, North M F and Cleland D J 2006 A new approach to modeling the effective thermal conductivity of heterogeneous materials Int. J. Heat Mass Transfer 49 3075-83

[31] Thovert J F, Wary F and Adler P M 1990 Thermal conductivity of random media and regular fractals J. Appl. Phys. 68 3872-83

[32] Bakker K 1997 Using the finite element method to compute the influence of complex porosity and inclusion structures on the thermal and electrical conductivity Int. J. Heat Mass Transfer 40 3503-11

[33] Zhang D X 2002 Stochastic Method for Flow in Porous Media (London: Academic)

[34] Kulasiri D and Verwoerd W 2002 Stochastic Dynamics Modeling Solute Transport in Porous Media (New York: Elsevier)

[35] Shoshany Y, Prialnik D and Podolak M 2002 Monte Carlo Modeling of the thermal conductivity of porous cometary ice Icarus 157 219-27

[36] Barta S and Dieska P 2002 Effective thermal conductivity of particulate composite materials Kovove Materialy-Metallic Materials 40 99-112

[37] Zhang H F, Ge X S and Ye H 2005 Effectiveness of the heat conduction reinforcement of particle filled composites Modelling Simul. Mater. Sci. Eng. 13 401-12
[38] Zhang H F, Ge X S and Ye H 2006 Randomly mixed model for predicting the effective thermal conductivity of moist porous media J. Phys. D: Appl. Phys. 39 220-26

[39] Qian J Y, Li Q, Yu K and Xuan Y M 2004 A novel method to determine the effective thermal conductivity of porous materials Sci. China, Ser. E 47 716-24

[40] Wang M, Wang J K, Pan N and Chen S Y 2007 Mesoscopic predictions of the effective thermal conductivity of microscale random porous media Phys. Rev. E at press

[41] Wang J K, Wang M and Li Z X 2006 A lattice boltzmann algorithm for fluid-solid conjugate heat transfer Int. J. Thermal Sci. at press (doi: 10.1016/j.thermalsci.2006. 04.012)

[42] Chen S Y and Doolen G D 1998 Lattice Boltzmann method for fluid flows Annu. Rev. Fluid Mech. 30 329-64

[43] Raabe D 2004 Overview of the lattice Boltzmann method for nano- and micorscale flow dynamics in materials science and engineering Modelling Simul. Mater. Sci. Eng. 12 R13-46

[44] Wang J K, Wang M and Li Z X 2006 Lattice Poisson-Boltzmann simulations of electro-osmotic flows in microchannels J. Colloid Interface Sci. 296 729-36

[45] Raabe D 2004 Overview of the lattice Boltzmann method for nano- and micorscale flow dynamics in materials science and engineering Modelling Simul. Mater. Sci. Eng. 12 R13-46

[46] Kang Q J, Zhang D X, Lichtner P C and Tsimpanogiannis I N 2004 Lattice Boltzmann model for crystal growth from supersaturated solution Geophys. Res. Lett. 31 L21604

[47] Doolen G D 1990 Lattice Gas Methods for Partial Differential Equations (New York: Addison Wesley)

[48] Succi S 2001 The Lattice Boltzmann Equation for Fluid Dynamics and Beyond (London: Oxford Science Press)

[49] He X Y, Chen S Y and Doolen G D 1998 A novel thermal model for the lattice Boltzmann method in incompressible limit J. Comput. Phys. 146 282-300

[50] Peng Y, Shu C and Chew Y T 2003 Simplified thermal lattice Boltzmann model for incompressible thermal flows Phys. Rev. E 68026701

[51] Peng Y, Shu C and Chew Y T 2003 A 3D incompressible thermal lattice Boltzmann model and its application to simulate natural convection in a cubic cavity J. Comput. Phys. 193 260-74

[52] D'Orazio A and Succi S 2003 Boundary conditions for thermal lattice Boltzmann simulations Lect. Notes Comput. Sci. 2657 977-86

[53] Zou Q S and He X Y 1997 On pressure and velocity boundary conditions for the lattice Boltzmann BGK model Phys. Fluids 9 1591-8

[54] Coveney P V, Maillet J B, Wilson J L, Fowler P W, Al-Mushadani O and Boghosian B M 1998 Lattice-gas simulations of ternary amphiphilic fluid flow in porous media Int. J. Modern Phys. C 9 1479-90

[55] Meakin P 1998 Fractals, Scaling and Growth Far from Equilibrium (Cambridge: Cambridge University Press)

[56] Wang M and Li Z X 2004 Numerical simulations on performance of MEMS-based nozzles at moderate or low temperatures Microfluidics and Nanofluidics $162-70$

[57] Guo Z Y and Li Z X 2003 Size effect on single-phase channel flow and heat transfer at microscale Int. J. Heat Fluid Flow 24 284-98

[58] Wang M and Li Z X 2004 Three-dimensional effect of gas flow in micro channels J. Eng. Thermophys. 25 840-2

[59] Kirkpatrick S 1973 Percolation and Conduction Rev. Mod. Phys. 45 574-88

[60] Li D Y 2006 Personal Communications, Department of Mechanical Engineering, Vanderbilt University

[61] Fukusako S 1990 Thermophysical properties of ice, snow and sea ice Int. J. Thermophys. 11 353-72

[62] Carson J K, Lovatt S J, Tanner D J and Cleland A C 2005 Thermal conductivity bounds for isotropic, porous materials Int. J. Heat and Mass Transfer 48 2150-8 